\title{
AUTOMOBILE THEFT RECOVERY
}

\author{
Ankit Yadav, Ankit Kumar Bharti, Bhawna Parashar, Avisha Sharma \\ Department of CSE \\ IMSEC, AKTU, Ghaziabad \\ Uttar Pradesh, India
}

\begin{abstract}
If we look around present world, we can easily find Global Positioning System (GPS) being used in several areas. A real time automobile theft recovery system using various (GPS, GSM, Arduino etc.) technologies has been proposed in this paper. Through this project the location of the vehicle, which has the device installed, is easy to track down. Soon after the location of the vehicle has been tracked, the data can be seen in the form of latitude and longitude coordinates through a text message on the user's mobile as well as the coordinates can be seen on the Android app too, as requested by the user. Initially, the inputs from the satellite are obtained by GPS installed in the device and are stored in the microcontroller's buffer. The coordinates are now stored in the microcontroller, so when the user requests the location of the vehicle then instantly the device is so designed to send a message having the coordinates to the registered user. As soon as the work of GSM is completed (which is after sending a text to the user) it gets deactivated. Then the GPS is activated again for the rest of the process. The android app can also be used to turn the engine ON/OFF and also change the mode from AUTO to MANUAL or vice versa. The hardware part that has been used in the project comprises of GPRS, GSM module, LCD to view the coordinates, ATMega Microcontroller MAX 232, Arduino, RS232 and relay.
\end{abstract}

Keywords- GPS, GSM, Arduino, ATMega Microcontroller, Android.

\section{INTRODUCTION}

The GPS GSM primarily based system as the name suggests combines GPS and GSM technologies. It can be widely employed in several applications and scores of users can be benefitted by it every day. The system is principally meant to extend the safety of vehicles and safety of people as well amongst the transit. This device installed in automobile is used to forestall stealing or to keep track of the vehicle's route. If the automobile is stolen, the system can send the location which will facilitate to find the automobile through the application. The following device can send the coordinates of almost any place where the vehicle might be at and performs expeditiously in areas having higher mobility. This paper describes Automobile theft recovery, that is employed to grasp the situation of the automobile using techniques like the Global Positioning System (GPS) and Global System for mobile communication (GSM). The salient characteristics of the system is that it is projected to use a development board, which can have GPS and GSM module seemingly like a single module which are firmly joined with a microcontroller. Alongside that, it'll conjointly magnify the sturdiness of the whole system. The ATMega328 microcontroller can give the interfacing to varied hardware peripherals to grasp the location of car. The Android user should click on the Vehicle location button which is present within the app. The messages are going to be mechanically sent to the number registered with the device. The system can respond by presenting the latitudes and longitudes (sensed by the GPS module) of the automobile to the registered android user.

\section{LITERATURE REVIEW}

Contrast of the prevailing tracking systems with the android app-based automobile theft recovery The prevailing tracking devices put in within the vehicles permits you to grasp the situation of the automobile. The GPS network used in the systems includes of twenty-seven international point satellites. In every of the vi orbital planes, there are 4 satellites, that make sure that they cowl the whole world. Every satellite sends a radio emission, that is handled by the receiver within the automotive. A minimum of 3 or 4 satellites are needed to grasp the location of the entered destination. This technique is understood as trilateration. But the prevailing GPS navigation system present within the cars doesn't furnish the owners much just in case he/she desires to know the position of the vehicle. This is where automobile theft recovery comes for the rescue. This technique is designed for all 
the vehicles and also the users having android phones. The android is the most generally used mobile Operating System. Due to its convenience and recognition amongst the users we've developed the system for android users. The user interface of the application is simple and easy to grasp. The owner simply should click the button so as to locate the position of the automobile. On clicking the button, text message is to be sent to the GSM modelled within the device. Then the hardware installed will send the coordinates to the user's number. Once the message is received, the whereabouts of the vehicle is notable. This device can facilitate the owner to invariably keep a watch on its vehicle. The user can also Switch Off/On the engine of the vehicle with the help of the app. It can also be converted into a tracking system with help of auto mode provided in the app.

\section{PROPOSED TECHNIQUE}

\section{Block Diagram}

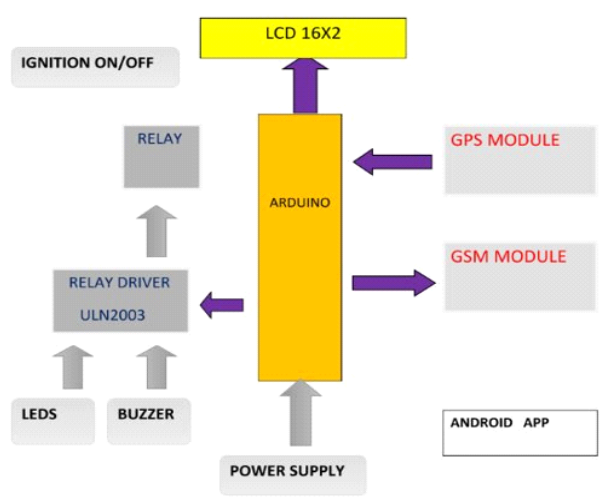

Fig1. Block Diagram of the System

\section{Illustration of Block Diagram}

The microcontroller 8052 is employed for interfacing the hardware peripherals. There's serial interfacing between the 8052 microcontroller and also the GSM electronic equipment and GPS receiver. The operations of the system are performed after taking the data from the GPS. This data is then sent to the RS232. It's then sent to MAX232 where the information is arranged, so it could be sent to the receiver pin $(\mathrm{Rx})$ of the microcontroller. After this, the information kept within the microcontroller buffer is sent to the MAX232 via the Lone-Star State (Transmitter pin).
The information is distributed into GSM via the RS232 protocol by MAX232. On demand, which is made by pressing the Vehicle location button within the android app, the location of the vehicle is sent from device to the registered user's mobile. The code for the Arduino is written, tested and debugged in Arduino IDE. For the Processing programming language and the Wiring project, this IDE (Integrated Development Environment) is used.

\section{FUNCTIONALITIES AND SUMMARY OF THE SYSTEM}

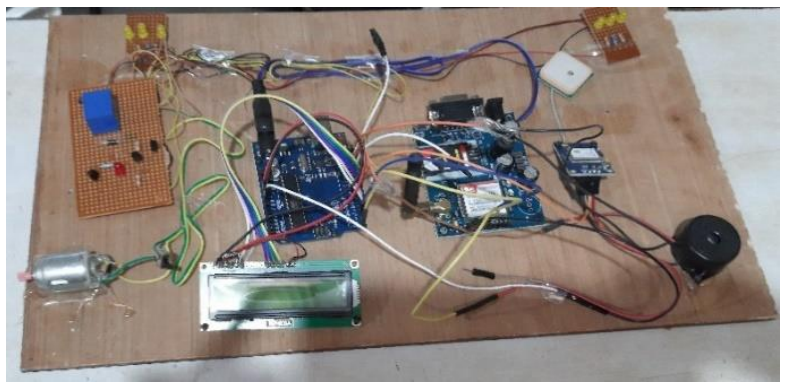

Fig 2. Hardware of the device

\section{GPS}

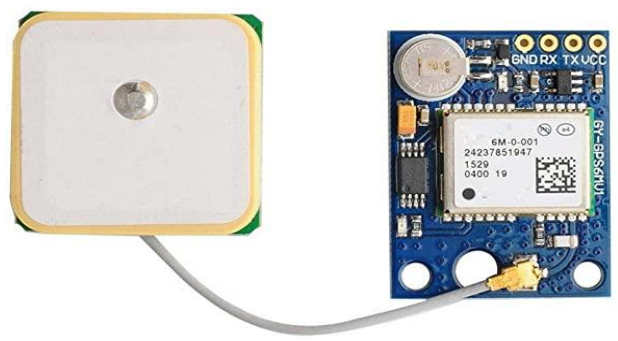

Fig3. GPS module

Global Positioning System (GPS) is a navigation system that uses signals from satellites to determine the location of a receiver. GPS satellites constantly send out a signal that contains their position and the exact time. A GPS receiver captures the signal from at least four satellites. It then determines its own coordinates by taking into account the time it took to receive the signal. The receiver's location can then be shown on a moving map display or sent to some other system. 
GSM

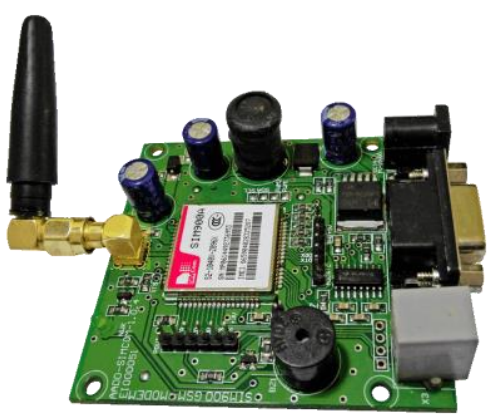

Fig4. GSM module

Digital cellular technology like GSM (Global System for Mobile Communication) is used to transmit mobile data as well as voice services. GSM technology was developed as a digital system using the time division multiple access (TDMA) technique for communication purposes. A GSM digitizes and reduces the data, then sends it down through a channel with two different streams of client data, each in its own particular time slot.

\section{Microcontroller}

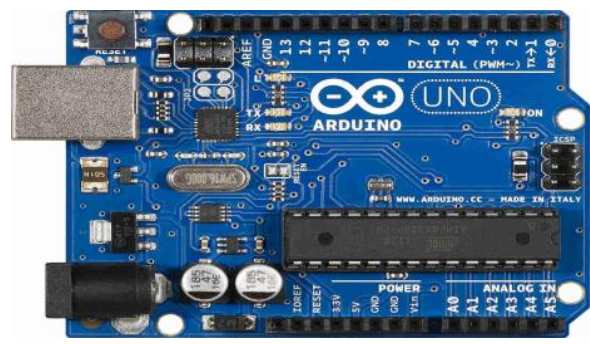

Fig5. Microcontroller

ATmega328P is one of the high performances AVR technology microcontroller with a large number of pins and features. It is designed by 8-bit CMOS technology and RSIC CPU which enhance its performance and its power efficiency get improved by auto sleeps and internal temperature sensor. This ATmega328P IC comes with internal protections and multiple programming methods which helps the engineers to priorities this controller for different situations. The IC allows multiple modern era communications methods for other modules and microcontrollers itself, which is why the microcontroller ATmega328P usage has been increasing every day.
MAX232

Max 232 is an ic(integrated circuit) which converts TTL(Transistor Transistor logic) logic level signal in to its equivalent RS-232c level signal and Rs-232c level to its equivalent TTL level signal.This ic is very important in case when we need to make connection and transfer data between devices that works on different signal level wave forms(TTL, Rs232c etc).

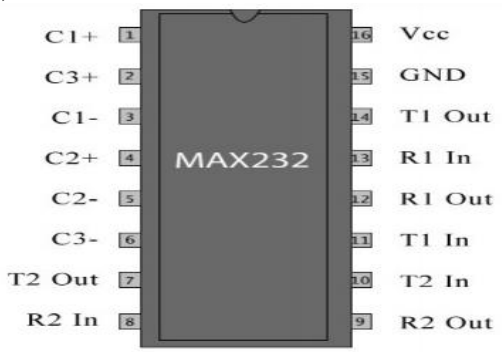

Fig6. MAX232

\section{RS232}

In RS232, 'RS' stands for Recommended Standard. It defines the serial communication using DTE and DCE signals. Here, DTE refers to Data Terminal Equipment and DCE refers to the Data Communication Equipment. The working of RS-232 can be understood by the protocol format. As RS-232 is a point-to-point asynchronous communication protocol, it sends data in a single direction. Here, no clock is required for synchronizing the transmitter and receiver. The data format is initiated with a start bit followed by 7-bit binary data, parity bit and stop bit which are sent one after another.

\section{Arduino IDE}

The code for the Arduino is written, tested and debugged in Arduino IDE. For the Processing programming language and the Wiring project, this IDE (Integrated Development Environment) is used.

\section{USABILITY}

It is terribly easy. So as to understand the vehicle's location, all a user must do is to press a button on the android app and consequently will switch the engine on/off. From the security point of view, it's quite secure as solely the location in the form of coordinates of the automobile is sent to the number of the registered user. 


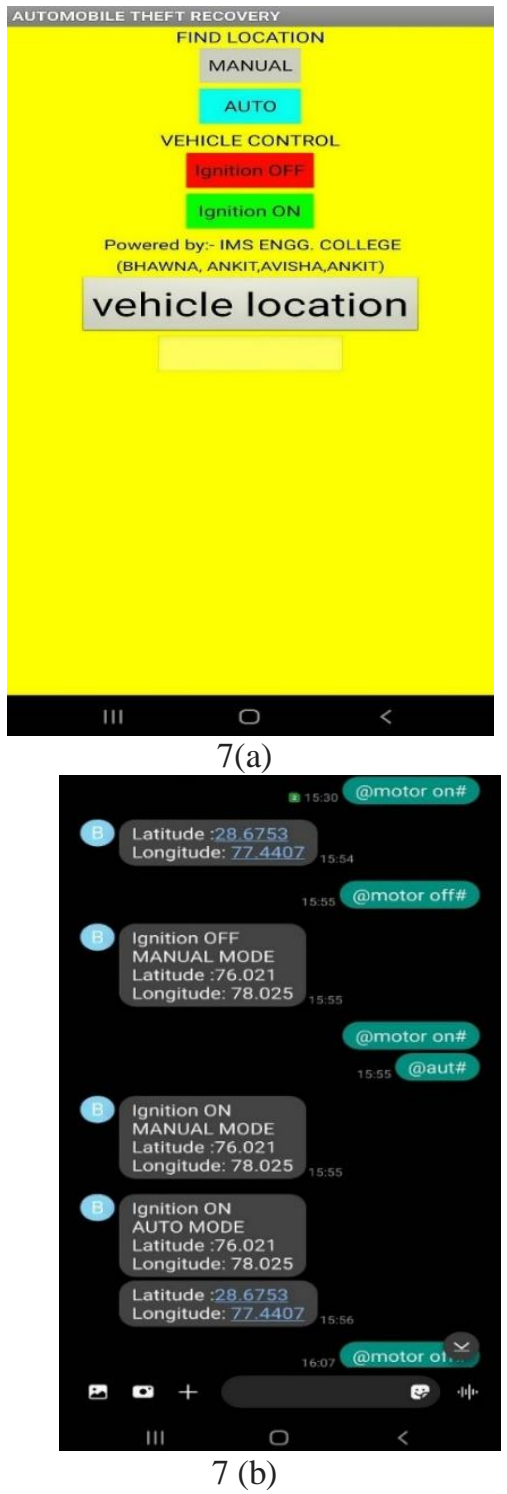

Fig7. (a) Screenshot of the Android App (b)Screenshot of Text message

\section{APPLICATIONS}

It may be utilized in variety of areas:

1. The most users of the merchandise can be of the transportation firms. Firms and the other emerging companies that provide cab services can make use of it. They just have to fixate the device in their vehicles to ensure the safety of commuters and their cabs.

2. Schools and Colleges may additionally install the device in their transportation that will help them keep an eye on the transport's route. This in turn will aid student's safety and security.
3. It can be utilized at Bus stands to effectively foresee and show the temporal order of the arrival of buses.

\section{CONCLUSION}

In the present world, the safety of the vehicles is of utmost priority as their security is at stake. The occurrences of stealing have become more prominent with time. This drives the patron to put in a pursuit device, which can facilitate them to understand the placement of their vehicle, which is maybe stolen. The automobile theft recovery system facilitates the user to track and monitor the location of his/her vehicle in real time. The merchandise developed is easy for anyone to use with a touch of information of smartphone. It's simple to install the system within the vehicle. Also, the device is easy to upkeep and scale of the device is compact since the GSM and GPS modules can be seen as a single module.

\section{REFERENCES}

[1] Ramani R., Valarmathy S., Dr. Suthanthira N., Selavaraju S., Thiruppathi M., Thagam R. (2013) "Vehicle Tracking and Locking Based GSM and GPS", (DOI: 10.5815/ijisa.2013.09.10)

[2] Franczyk M. F., and Vanstone D. J. (2008) "Vehicle Warning System", Patent number: 7362239. [3] Stalling W. (2005) "Wireless Communication and Networks", 2nd edition, prentice hall of India

[4] Kant K. (2007) "Microprocessor and Microcontroller", Eastern Company Edition, New Delhi

[5] Maurya K., Singh M., Jain N., (2012) "Real Time Vehicle Tracking System using GSM and GPS technology- An Antitheft Tracking System", International Journal of Electronics and Computer Science Engineering, page no. 1103-1107

[6] Song Kai-Tai, Yang Chih-Chieh (2005) "Front Vehicle Tracking Using Scene Analysis", Proceedings of the IEEE International Conference on Mechatronics \& Automation.

[7] Gaonkar S. R., "Microprocessor Architecture Programming and Application", Wiley Eastern Limited, New Delhi

[8] El-Medany W., Al-Omary A., Al-Hakim R., AlIrhayim S., Nusaif M., (2010) "A Cost-Effective RealTime Tracking System Prototype Using Integrated GPS/GPRS Module, "Wireless and Mobile Communications (ICWMC), $6^{\text {th }} \quad$ International Conference, page no. 521-525 
[9] Fleischer P.B., Nelson A.Y., Sowah R.A., Bremang A., (2012) "Design and development of GPS/GSM based vehicle tracking and alert system for commercial inter-city buses, "Adaptive Science \& Technology (ICAST), 2012 IEEE $4^{\text {th }}$ International Conference page no. 1-6

[10] Le-Tien T., Phung Vu (2010) - "The Routing and Tracking System for Mobile Vehicles in Large Area," Electronic Design, Test and Application, 2010. DELTA '10. Fifth IEEE International Symposium, page no. 297-300.

[11] Khan Abed M.E.(Student), Mishra R., (2012) "GPS - GSM Based Tracking System" SSCET, CSVTU, Bhilai, India International Journal of Engineering Trends and Technology- vol.3, page no. 161-164.

[12] El-Medany W.M., Alomary A., Al-Hakim R., AlIrhayim S., Nousif M., (2010) "Implementation of GPRS- Based Positioning System Using PIC Microcontroller, "Computational Intelligence, Communication Systems and Networks (CICSN), Second International Conference, page no. 365-368. [13] Lita I., Cioc I.B., Visan D.A., (2006) "A New Approach of Automobile Localization System Using GPS and GSM/GPRS Transmission," Electronics Technology, 29th International Spring Seminar, page no.115-119. 